\title{
Hospital variations during the first year of the COVID-19 pandemic in a referral hospital in a low-to- middle-income country: A large single-center cohort study
}

Carmelo Dueñas-Castell

Clínica Gestión Salud

Wilfrido Coronell

Clínica Gestión Salud

Diana Borre-Naranjo

Clínica Gestión Salud

Amilkar Almanza

Clínica Gestión Salud

Leydis Lora

Clínica Gestión Salud

Rafael Navarro

Clínica Gestión Salud

Jose Rojas-Suarez ( $\square$ joseantonio.rojas.suarez@gricio.com )

Universidad de Cartagena

Research Article

Keywords: COVID-19, RT-PCR, hospital admission

Posted Date: September 2nd, 2021

DOI: https://doi.org/10.21203/rs.3.rs-863006/v1

License: (c) (1) This work is licensed under a Creative Commons Attribution 4.0 International License.

Read Full License 


\section{Abstract \\ Background}

COVID-19 infection is characterized by its heterogeneity in clinical presentation. Hospital admission, intensive care unit (ICU) admission and mortality rates have shown wide variations throughout the months. Colombia experienced a high rate of COVID-19 infection, leading to significant morbidity and mortality. To date, there are only a few studies reporting peak variations. Therefore, we aimed to explore the potential reasons for epidemiological changes in COVID-19 with time.

\section{Methods}

This study was conducted at a tertiary referral center in Cartagena, Colombia, between March 7, 2020, and February 28, 2021. We included patients aged $>18$ years with suspected or confirmed COVID-19. A diagnosis was made using the reverse-transcriptase polymerase chain reaction (RT-PCR) test. We extracted information from the Colombian National Surveillance System (SIVIGILA). We collected demographic data, such as age, sex, RT-PCR test results, and information on close contacts (awareness of the disease). We categorized the study period into two according to the two peaks defined in Colombia: the first period included weeks 10-35 (March 7-August 30, 2020), and the second period included weeks 36-60 (September 1, 2020-February 28,2021 ). We adjusted the weekly overall mortality by covariates to identify potential changes during the pandemic.

\section{Results}

In total, 2761 COVID-19 patients were hospitalized at our institution. The most significant number of cases occurred in the first and second periods during week 21 (peak p $_{1}: 121$ patients) and week 50 (peak : $^{2}$ 128 patients), respectively.

\section{Background}

Coronavirus disease (COVID-19) is characterized by heterogeneity in its clinical presentation(1). Some patients are asymptomatic, while others experience severe clinical symptoms with a fatal outcome. Severe cases lead to hospitalization, with rapid progression to hypoxia, respiratory distress, and the need for oxygen or ventilatory support(1).

Hospital admission, ICU admission, morbidity, and mortality rates have shown wide variations throughout the months, with different trends in the pandemic and between countries(1)(2). The pandemic led to an unprecedented scientific response. In parallel with infections and deaths, the medical world released an enormous amount of information on the diagnosis and management of COVID-19(2). 
Colombia experienced a high rate of COVID-19 infection, leading to significant morbidity and mortality rates. The increased number of hospitalizations associated with COVID-19 peaks has generated significant challenges in the adequacy of hospital infrastructure, allocation of human resources, personal protective equipment (PPE), pharmacological and non-pharmacological strategies. Humankind learned a lot from previous pandemics, and the experience accumulated during the last year will generate valuable information to respond to future crises(3)(4). Even with this experience, to what extent this situation affects different outcomes is largely unknown, and to date, there are only a few studies describing peak variations. Thus, we aimed to explore potential reasons for epidemiological changes over time by evaluating patient characteristics and institutional protocol adjustments.

\section{Materials And Methods}

\section{Study design and population}

We conducted this retrospective cohort study at the tertiary referral center in Cartagena, Colombia, between March 7, 2020, to February 28, 2021. We included patients older than 18 years old with clinical signs or confirmed COVID-19. COVID-19 was diagnosed according to the National Institute of Health Guidelines. We did not exclude any patients from this study. Given the retrospective data collection, based on the data in the medical records, we received approval and informed consent waiver from the Gestion Salud Clinic Institutional Review Board before starting the study.

\section{Institutional protocol}

The emergency department (ED) designed an admission protocol for patients with respiratory symptoms, including diagnosing COVID-19 infection using RT-PCR test in orotracheal/nasopharynx aspirate based on international and national guidelines. The institutional medical team also defined the severity of illness upon admission and decided the patient allocation (hospital admission or outpatient management) based on the magnitude of respiratory symptoms and some prognostic scores (NEWS2, CALL, CURB-65 scores)(5)(6).

\section{Institutional changes}

The COVID-19 pandemic caused significant challenges with the adequacy of infrastructure in allocating human resources, PPE, and pharmacological and non-pharmacological interventions.

The institution expanded the total capacity of intensive care beds for COVID-19 patients. By April 2020, the institutional advisory board (IAB) transformed the intermediate care area into an ICU and the operating room into a respiratory care area. All external consultation services, including offices and waiting halls, were transformed into a large emergency area adapted for respiratory consultation. The new emergency area was equipped with oxygen supplies, mechanical ventilators, and monitors (Additional file 1).

\section{Staff training}


By February 2020, the IAB trained all the institutional staff on the use of COVID-19 guidelines. Subsequently, The IAB assigned shifts to the healthcare staff to cover the different areas of the institution.

\section{Guidelines Audit and review}

The IAB created a multidisciplinary team with an infectious disease specialist (WC) and a senior critical care physician (CDC). This team focused on audit, revise, and adjust national and international evidence in the local healthcare system, to develop a rationale for the current and emerging literature and turn it into a hospital guideline. The IAB only approved treatments and interventions with established mortality benefits for COVID-19 based on randomized controlled trials or well-conducted observational studies after a unanimous decision. At the time of this study, the only treatment approved for COVID-19 patients was corticosteroids.

\section{Variables and data collection}

We obtained information from the Colombian National Surveillance System (SIVIGILA, Sistema de Vigilancia en Salud Pública) (SIVIGILA) database. In addition, we collected demographic data such as age $(<50,50-69,70-79$, and $>80$ years), gender (male and female), RT-PCR results (positive or negative), information on close contact (disease awareness) and hospital service area where patients were treated (outpatient, emergency room, general ward, or ICU). The primary exposures of interest included the duration (wk.) of patient consultation to the hospital, based on the International Organization for Standardization date week (ref: http://myweb.ecu.edu/mccartyr/isowdcal.html).

\section{Comparison between periods}

To facilitate comparison across time, we categorized time into two equal periods according to the two peaks defined in Colombia: (First period: March-August 2020; second period: September 2020- February 2021); thus, the first period included weeks 10-35 [from March 7 to August 30, 2020]; and the second period had weeks 36-60 [from September 1, 2020, to February 28, 2021]. We considered a total period of 12 months for data extraction. We described the epidemic curves, including periods and peaks, using four indices: the duration (weeks) of the onset (or new onset in the case of the second period) to peak (DOP), the duration from the onset to the end (DOE), the DOP/DOE ratio, and the steepness of epidemic curves, expressed as steepness index (SI) (peak number/DOE)(7)

We divided patients into two groups (survivors and non-survivors) according to the presence of the outcome (weekly in-hospital mortality at 30 days of follow-up). Other outcomes of interest were demographic changes in patients' characteristics (gender, RT-PCR results, and disease awareness, defined as patient recognition of close contact, and knowledge of COVID-19, comprising associated conditions or treatments). In addition to the overall mortality analysis with complete cases, we proposed a sensitivity analysis during the peak period called the "worst time" for a pandemic (with an expected saturation of hospital beds, supplies shortages, personnel exhaustion, etc.). We consider the "worst time" as the beforeafter $50 \%$ decline in the number of cases per week around the peak period. 


\section{Statistical analysis:}

We reported descriptive analyses as median and interquartile ranges for continuous data and as proportions and percentages for categorical data. We conducted a univariate analysis of continuous data using the Rank-Sum test analysis. Categorical variables were analyzed using the $\chi^{2}$ or Fisher's exact test. We stratified the number of patients admitted each week and the weekly overall mortality by covariates (male sex, positive RT-PCR result, and disease awareness) to identify potential changes during the pandemic.

A forward logistic regression model was used to explore whether all exposure variables were associated with the primary outcome. A marginal effect model was used to describe the average effect of change in each week (stratified by covariates) on the difference in the probability of mortality. A multivariable logistic regression model was used to calculate the OR and confidence intervals at the $95 \%$ level $(95 \% \mathrm{Cl})$ for mortality. We performed all analyses and graphics using GraphPad Prism version 9.1.2 for Mac, GraphPad Software, San Diego, California USA, www.graphpad.com; and STATA software, version 15.0 (Stata Corp., College Station, TX). A p-value $<0.05$ was considered significant. We wrote this report following the Enhancing the Quality and Transparency of Health Research (EQUATOR) STROBE guidelines.

\section{Results}

In total, 2761 patients were hospitalized with clinical signs or confirmed COVID-19 from March 2020 to February 2021, representing 6.95\% of all admission during the period (2761/39696). Among these, 1455 were hospitalized during the first period and 1306 in the second. During the first period, the most significant number of severe acute respiratory infections (peak ${ }_{1}$ ) occurred in week 21 (from May 22 to 28, 121 patients). During the second period, the higher number of cases occurred in week 50 (peak ${ }_{2}$ ) (from December 11 to 17,128 cases). The DOP from the first period was 6 weeks and that from the second period was 10 weeks. The DOE was 18 weeks for the first period and 19 weeks for the second period. The DOP/DOE ratio was 0.33 and 0.5 for the first and second periods, respectively. Finally, the SI for the first period was 6.9 versus 6.7 for the second (Figure 1). Based on peak ${ }_{1}$ and peak ${ }_{2}$, a before-after $50 \%$ decline in weekly cases was identified from week 19 to 30 during the first period (worst time ${ }_{1}$ ) and from week 47 to 56 during the second period (worst time ${ }_{2}$ ).

In total, 287 cases (19.8\%) were managed in the general ward during the first period, compared to 317 $(24.2 \%)$ during the second period (percentage of change 10.4\%; $p$-value 0.005$)$. There were no differences in ICU admission rates between periods (Table 1, Figure 2). The highest hospital admission rate was observed between weeks 20-30 (519 patients, $66.4 \%$ ) for the first period and between weeks 50-56 (332 patients, $46.3 \%$ ) for the second period. The proportion of patients admitted to ICU peaked at week 30 (27 patients) during the first period and at week 55 (30 patients) in the second period (Figure 2). 
Table 1. Demographic data of patients with acute respiratory symptoms in our institution from March 2020 to February 2021 during the first and second periods.

\begin{tabular}{|c|c|c|c|c|}
\hline Variable & Total & $\begin{array}{l}\text { First period } \\
\text { (Week 10-35) }\end{array}$ & $\begin{array}{l}\text { Second period } \\
\text { (Week 36-60) }\end{array}$ & $p$-value \\
\hline & $(n=2761)$ & $(n=1451,52.6 \%)$ & $(n=1310,47.4 \%)$ & \\
\hline \multicolumn{5}{|l|}{ Demographics } \\
\hline Age (median, IQR) & $49(34-67)$ & $49(35-66)$ & $48(33-67)$ & 0.44 \\
\hline$<50(\mathrm{n}(\%))$ & $1416(51.3)$ & 734(50.4) & $682(52.2)$ & 0.35 \\
\hline $50-69(n(\%))$ & $781(28.3)$ & $438(30.1)$ & $343(26.3)$ & 0.02 \\
\hline 70-79 (n(\%)) & $304(11)$ & $156(10.7)$ & 148(11.3) & 0.60 \\
\hline$\geq 80(\mathrm{n}(\%))$ & $260(9.4)$ & $127(8.7)$ & 133(10.2) & 0.19 \\
\hline positive RT-PCR (n(\%)) & $841(30.5)$ & $354(24.4)$ & $487(37.2)$ & 0.001 \\
\hline Male sex $(n(\%))$ & $1526(55.3)$ & $839(57.8)$ & $689(52.6)$ & 0.008 \\
\hline Disease awareness $(\mathrm{n}(\%))$ & $597(21.6)$ & 263(18.1) & $334(25.5)$ & 0.001 \\
\hline \multicolumn{5}{|c|}{ Hospital characteristics (n(\%)) } \\
\hline General ward & $604(21.9)$ & 287(19.8) & $317(24.2)$ & 0.005 \\
\hline Intensive care unit & $565(20.5)$ & $301(20.7)$ & $264(20.2)$ & 0.7 \\
\hline Emergency Room & $335(12.1)$ & 199(13.7) & $136(10.4)$ & 0.007 \\
\hline Outpatients & $1257(45.5)$ & $664(45.8)$ & $593(45.3)$ & 0.79 \\
\hline Overall mortality (n(\%)) & $336(12.2)$ & $200(13.8)$ & $136(10.4)$ & 0.006 \\
\hline
\end{tabular}

Abbreviation: IQR, interquartile range; RT-PCR, reverse-transcriptase polymerase chain reaction.

The median age was 49 years (IQR 34-66). Most patients were younger than 50 years (1416/ (51.3\%), with an increased number of patients between aged 50-69 years during the first period (438[30.1\%] vs. 343[26.3]) (Table 1, Figure 3A). The comparison of the number of outpatients between periods stratified by age showed an increased probability of being an outpatient among patients aged less than 50 years (marginal effect 69.1\%, 95\% Cl 66.7- 71.5), compared to patients older than 50 years (marginal effect 20.6\%, 95\% Cl 18.5-22.8), without differences between periods (Figure 3B). In contrast, among in-hospital patients, the probability of being hospitalized was higher in patients aged 70-79 years (marginal effect 
$46.4 \%, \mathrm{Cl} 95 \% 36.0-56.7 \%$ ) (Figure 3C). There was a lower probability of ICU admissions in people younger than 50 years (marginal effect $9.6 \%, 95 \% \mathrm{Cl}$ 8.14-11.2) than those older than 50 (marginal effect $31.8 \%, 95 \% \mathrm{Cl} 29.334 .2$ ). Additionally, the frequency of ICU admission among patients younger than 50 years decreased in the second period (Figure 3D).

The proportion of men admitted was higher (1526/2761 [55.3\%]), with a decrease from the first to the second period (839 [57.8\%] vs. 689 [52.6\%]). However, the proportions of men and women were similar in week 60 (Table 1, Figure 4A). Of all 2761 cases, 841 (30.5\%) had a positive RT-PCR result, with a difference between the two periods (354/1451[24.4\%] vs, 487/1310 [37.2\%]: percentage of change, 52.4\%; p-value 0.001) (Table 1, Figure 4B). Among patients reporting disease awareness, the mortality rate was lower across all periods (marginal effect among patients with disease awareness 4.2\%, 95\% Cl 2.65-5.94 vs. $14.2 \%, 95 \% \mathrm{Cl} 12.8-15.7$ among patients without disease awareness). Despite this, the mortality rate decreased in both groups of patients (with and without disease awareness) across the two periods (Table 1, Figure 4C).

The overall hospital mortality rate was $12.2 \%$ (336 patients), with a decline in the unadjusted mortality rate between the two periods (201 [13.8\%] from week $10-35$ vs. 135 [10.3\%] from week 36-60) (Table 1). Consistent with this result, the marginal analysis of mortality rate showed a decrease in overall mortality from 16.5\% (95\% Cl 13.3-19.8) in week 10 to 8.91 (Cl 95\% 6.95-10.8) in week 60 (p-value <0.001) (Figure 5). In the sensitivity analysis of the "worst time," the mortality rate during "worst time ${ }_{1}$ " (week 19 to 30) was higher than the mortality rate during the "worst time 2 " (week 47-56) (13\% [95\% Cl 11.8-16.8] vs. 9\% [95\% Cl 6.8-11.5]). There was a $28 \%$ reduction in the odds of overall mortality during the second period (odds ratio [OR] 0.72 [95\% $\mathrm{Cl} 0.57-0.91], \mathrm{p}<0.0001$ ). After adjustment for age, male sex, RT-PCR result, disease awareness, and ICU admission, the odds ratio of overall mortality during the second period was $0.70(0.5-0.91 ; p<0 \cdot 0001)$. However, overall mortality remained persistently high for patients admitted to ICU (42.9\% [95\% Cl -34.1-51.7] in the first period vs. 35.7\% [95\% Cl 27.8-43.7] in the second period); $\mathrm{p}$ value 0.33 .

\section{Discussion}

This study is the first Colombian report on the experience in a tertiary hospital during the first year of the COVID-19 pandemic, describing a decrease in the overall mortality associated with changes in epidemiological, clinical, and hospital characteristics across the time. We found more patients, predominantly men, with a higher frequency of emergency room allocation and higher mortality rate in the first period. On the other hand, we identified an increased disease awareness level in the second period, with a greater positivity rate in the RT-PCR result and higher hospitalization rates in the general ward. In addition, we found that age had a significant impact on mortality, with the likelihood of dying being 3.5 and 8.5 times higher among those between 61-80 and 81-99 years of age, respectively. According to published literature, age is associated with mortality in both periods, in line with the reports in other viral infections(8)(9)(10). 
Mortality from COVID-19 is variable in the literature(11)(12). Such variability has been attributed to geographical differences in the prevalence of comorbidities and the severity of the disease. Thus, the highest mortality rate has been reported in Latin America and Europe, where, in addition, older age and more comorbidities have been reported(13).

In this publication, as in the literature, the probability of ICU admission also increased with age. Recent reports suggest that approximately $14 \%-29 \%$ of hospitalized patients with COVID-19 pneumonia require intensive care. In these reports, the patients were older, predominantly male, and had underlying comorbidities(14).

An aspect that contributed to a more comprehensive, timely, and adequate management of patients was the improved performance of diagnostic tests. The rate of positive RT-PCR was higher in the second period. This change might be explained by three factors that improved molecular test performance compared to the first period-first, the inclusion of the Berlin protocol with better sensitivity(15)(16). Second, we optimized the "right time" in the disease course to perform the RT-PCR test and decreased false negatives. Finally, a change in the collected specimens, from the nasopharyngeal swab to tracheal aspiration, and bronchoalveolar washing, with higher sensitivity and/or higher viral load(17).

We also explored disease awareness in our study and observed that, initially, the attitude of the community was different and could impact the time to seek attention. History has shown that fear, discrimination, and stigmatization are frequently observed in a pandemic, especially in the first moments of disease onset(18). This situation can cause delays in consulting and seeking assistance, and worse outcomes, as was the case in our study. For this reason, it is essential to detect and prevent misinformation while educating and informing the community to obtain favorable results(19)(20)(21).

The epidemic curve ascent, that is, the speed with which the cases occurred during a peak, evaluated by DOP, DOE, DOP/DOE, and SI, could explain the higher mortality rate in the first pandemic period. These epidemiological indicators, proposed in other scenarios, have been little evaluated during the pandemic and could help decision-making(7).

Concomitantly, the relationship between the incidence of COVID-19, the frequency of ICU admissions, and the mortality rate has been raised(12). The entire world was isolated and confined for months trying to prepare ICU beds and ventilators to prevent the pandemic from overwhelming the capacity of health systems. Our study showed higher mortality and hospitalization rates in the emergency room during the first period. In contrast, we had a higher probability of hospitalization in the general ward during the second period, assuming a better patient allocation, with no differences in ICU admission between the periods.

The most important finding in our study was a progressive and significant decrease in mortality, with a lower risk of mortality for patients after week 35 . The need for emergency beds, general hospitalization, and intensive care vary with the dynamics of the pandemic, and their availability must also change. The ability to overcome these issues possibly affects clinical outcomes. An improvement in identifying and 
allocating patients according to severity using early warning systems and institutional response teams may optimize the available resources. These changes can facilitate an institution to face the pandemic, impacting clinical outcomes(12).

\section{Limitations}

The limitations of this study should be acknowledged. The retrospective single-center cohort analysis can make it difficult to generalize results in a broader context. However, the follow-up period of one year, the inclusion of two periods, and similarities in characteristics with those of other Latin American regions may help interpret this finding in a global context. Additionally, multicenter studies comparing institutional changes, differences, and similarities are needed to understand the shift in mortality during the COVID-19 pandemic.

\section{Clinical utility}

Although it is impossible to rule out the changes regarding the virus, our results allowed us to suggest, like other studies, that better knowledge, preparation, and availability of resources, both in the community and in the health services, can impact clinical outcomes. In addition, our results can help generate proposals to face the subsequent surges and/or future pandemics.

\section{Conclusion}

A famous aphorism attributed to the poet Archibald MacLeish claim that "there is only one thing more painful than learning from experience, and that is not learning from experience." Thus, our findings showed a lower mortality rate during the second period, possibly explained by a better recognition of the disease, performance, and availability of diagnostic tests, improved knowledge across the pandemic and led to a better administration of the health resources.

\section{Declarations}

\section{Ethics approval and consent to participate}

We received ethical approval from the Gestion Salud Clinic Institutional Review Board before starting the study.

\section{Consent for publication}

Not applicable

\section{Availability of data and materials}

The data used to support the findings of this study are restricted by the Institutional Review Board of Gestion Salud Clinic to protect patient privacy. Data are available from [Jose Rojas-Suarez, 
joseantonio.rojas.suarez@gricio.com] for researchers who meet the criteria for access to confidential data.

\section{Competing interests}

The authors have nothing to declare

\section{Funding}

This research was funded by the Gestion Salud Clinic.

\section{Authors' contributions}

Name: CDC

Contribution: This author conceived and designed this research; analyzed and interpreted the data; drafted the manuscript, critically revised it, and approved the final version.

Name: WC

Contribution: This author helped analyze the data and wrote and approved the final version of the manuscript.

Name: DBN

Contribution: This author analyzed the data and helped write and approved the final version of the manuscript.

Name: AA

Contribution: This author helped write and critically revised the manuscript and approved the final version.

Name: LL

Contribution: This author wrote and approved the final version of the manuscript.

Name: RN

Contribution: This author helped analyze and interpret the data and helped write and approve the manuscript's final version.

Name: JRS 
Contribution: This author conceived and designed this research; analyzed and interpreted the data; drafted the manuscript, critically revised it, and approved the final version.

\section{Acknowledgments}

The authors thank Nazly Villadiego and Elibeth Medina for their support during this project.

\section{Abbreviations}

COVID-19, coronavirus disease 2019; ICU, intensive care unit; RT-PCR, Reverse transcriptase-polymerase chain reaction; DOP, duration of the onset to peak; DOE, duration from the onset to the end; SI, steepness index; IAB, institutional advisory board; PPE, personal protective equipment; SIVIGILA, Colombian National Surveillance System

\section{References}

1. Li J, Huang DQ, Zou B, Yang H, Hui WZ, Rui F, et al. Epidemiology of COVID-19: A systematic review and meta-analysis of clinical characteristics, risk factors, and outcomes. J Med Virol. 2021;93:144958.

2. Mishra SK, Tripathi T. One year update on the COVID-19 pandemic: Where are we now? Acta Trop. 2021;214:105778.

3. Cook AH, Cohen DB. Pandemic Disease: A Past and Future Challenge to Governance in the United States. Rev Policy Res. 2008;25:449-71.

4. Baber R. Pandemics: learning from the past. Climacteric. 2020;23:211-2.

5. Saavedra Trujillo CH. Consenso colombiano de atención, diagnóstico y manejo de la infección por SARS-COV-2/COVID 19 en establecimientos de atención de la salud. Recomendaciones basadas en consenso de expertos e informadas en la evidencia. Infectio. 2020;24:1.

6. Accini Mendoza JL, Nieto Estrada VH, Beltrán López N, Ramos Bolaños E, Molano Franco D, Dueñas Castell C, et al. Actualización de la Declaración de consenso en medicina critica para la atención multidisciplinaria del paciente con sospecha o confirmación diagnóstica de COVID-19. Acta Colombiana de Cuidado Intensivo. 2020;20:1-112.

7. Saito S, Saito N, Itoga M, Ozaki H, Kimura T, Okamura Y, et al. Influence of Media on Seasonal Influenza Epidemic Curves. Int J Infect Dis. 2016;50:6-9.

8. Petrilli CM, Jones SA, Yang J, Rajagopalan H, O'Donnell L, Chernyak Y, et al. Factors associated with hospital admission and critical illness among 5279 people with coronavirus disease 2019 in New York City: prospective cohort study. BMJ. 2020;369:m1966.

9. Simonsen L, Fukuda K, Schonberger LB, Cox NJ. The impact of influenza epidemics on hospitalizations. J Infect Dis. 2000;181:831-7. 
10. Zhou H, Thompson WW, Viboud CG, Ringholz CM, Cheng P-Y, Steiner C, et al. Hospitalizations associated with influenza and respiratory syncytial virus in the United States, 1993-2008. Clin Infect Dis. 2012;54:1427-36.

11. Ji Y, Ma Z, Peppelenbosch MP, Pan Q. Potential association between COVID-19 mortality and healthcare resource availability. Lancet Glob Health. 2020;8:e480.

12. Immovilli P, Morelli N, Rota E, Guidetti D. COVID-19 mortality and healthcare resources: Organization. Medicina intensiva. 2020; doi:10.1016/j.medin.2020.05.014.

13. Thakur B, Dubey P, Benitez J, Torres JP, Reddy S, Shokar N, et al. A systematic review and metaanalysis of geographic differences in comorbidities and associated severity and mortality among individuals with COVID-19. Sci Rep. 2021;11:8562.

14. Suleyman G, Fadel RA, Malette KM, Hammond C, Abdulla H, Entz A, et al. Clinical Characteristics and Morbidity Associated With Coronavirus Disease 2019 in a Series of Patients in Metropolitan Detroit. JAMA Netw open. 2020;3:e2012270.

15. Cheng MP, Papenburg J, Desjardins M, Kanjilal S, Quach C, Libman M, et al. Diagnostic Testing for Severe Acute Respiratory Syndrome-Related Coronavirus 2: A Narrative Review. Ann Intern Med. 2020;172:726-34.

16. Corman VM, Landt O, Kaiser M, Molenkamp R, Meijer A, Chu DK, et al. Detection of 2019 novel coronavirus (2019-nCoV) by real-time RT-PCR. Euro Surveill. 2020;25:2000045.

17. Wang W, Xu Y, Gao R, Lu R, Han K, Wu G, et al. Detection of SARS-CoV-2 in Different Types of Clinical Specimens. JAMA. 2020;323:1843-4.

18. Chopra KK, Arora VK. Covid-19 and social stigma: Role of scientific community. Indian J Tuberc. 2020;67:284-5.

19. Ki M. Epidemiologic characteristics of early cases with 2019 novel coronavirus (2019-nCoV) disease in Korea. Epidemiol Health. 2020;42:e2020007.

20. Chersich MF, Gray G, Fairlie L, Eichbaum Q, Mayhew S, Allwood B, et al. COVID-19 in Africa: care and protection for frontline healthcare workers. Global Health. 2020;16:46.

21. van Halem K, Bruyndonckx R, van der Hilst J, Cox J, Driesen P, Opsomer M, et al. Risk factors for mortality in hospitalized patients with COVID-19 at the start of the pandemic in Belgium: a retrospective cohort study. BMC Infect Dis. 2020;20:897.

\section{Figures}




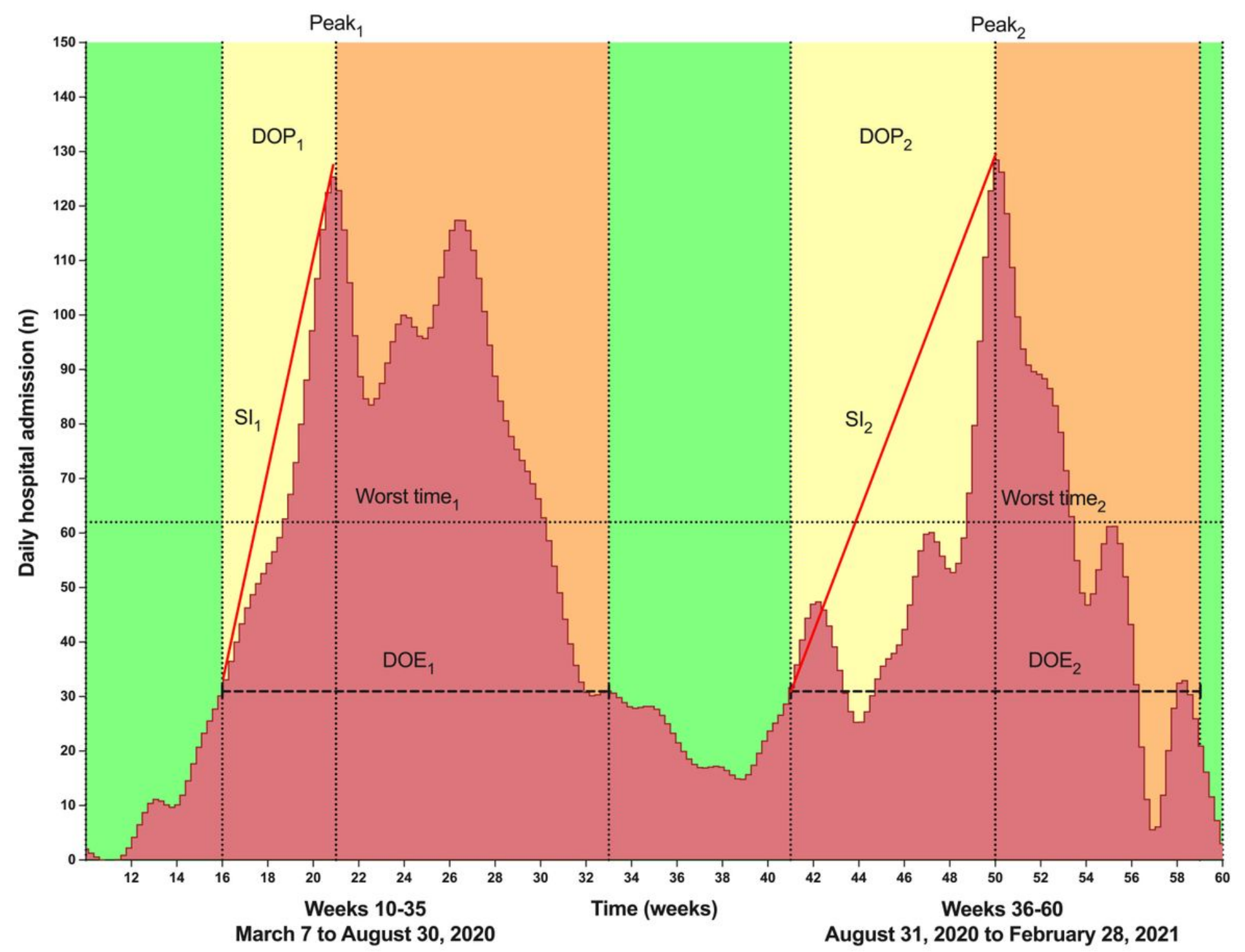

\section{Figure 1}

Daily respiratory cases during the first 12 months of the pandemic The first period occurred between March-August 2020 and the second period between September 2020-February 2021. 


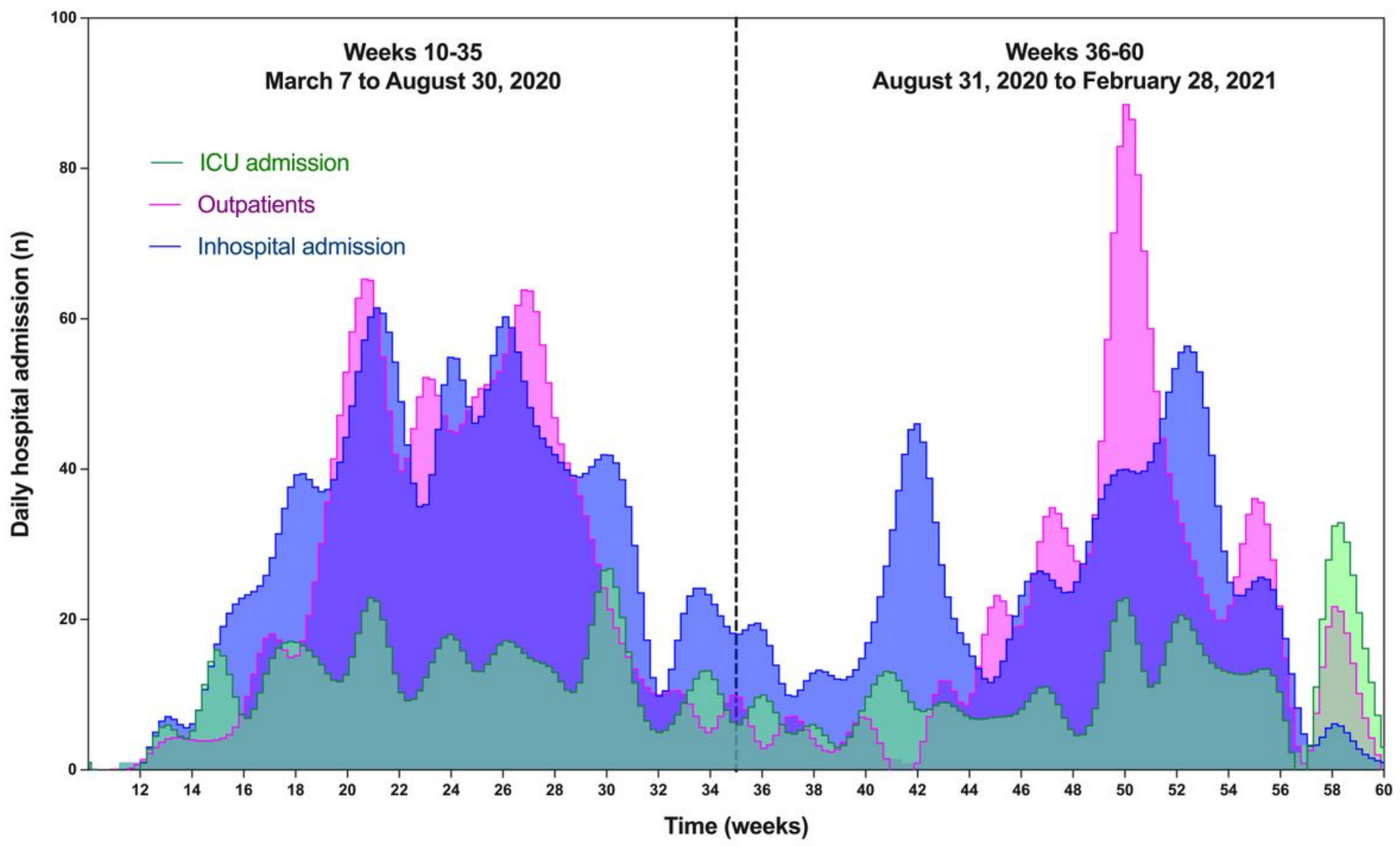

Figure 2

Daily COVID-19 cases during the first 12 months of the pandemic The number of patients is stacked by in hospital setting group. 
A

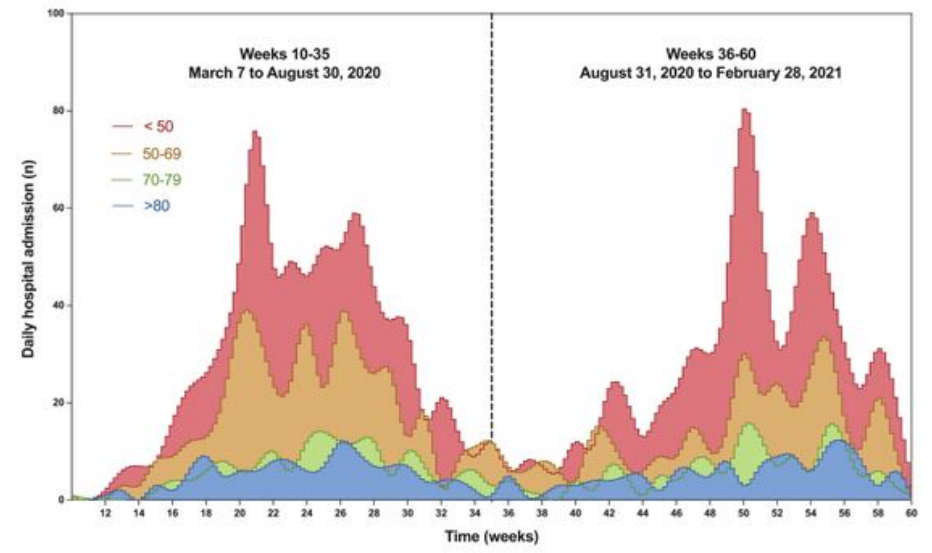

C

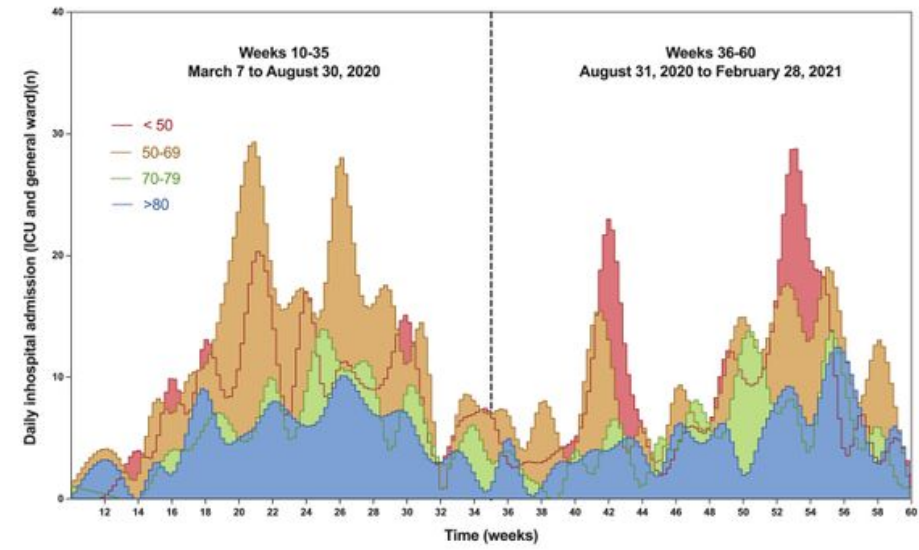

B

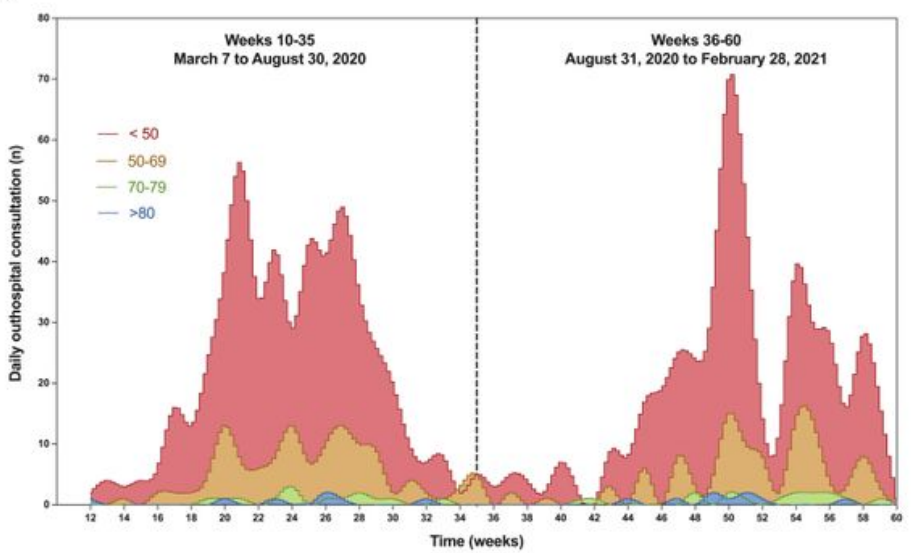

D

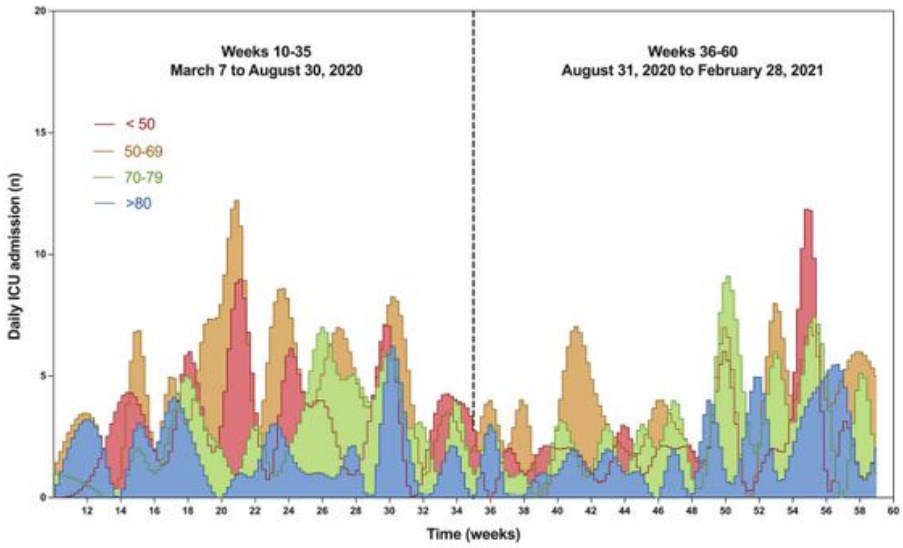

Figure 3

Daily adult COVID-19 admissions by age Cases are stacked by age group and A) all cases; B) outpatient consultation C) in hospital admissions (General ward and ICU) D) ICU admission. 

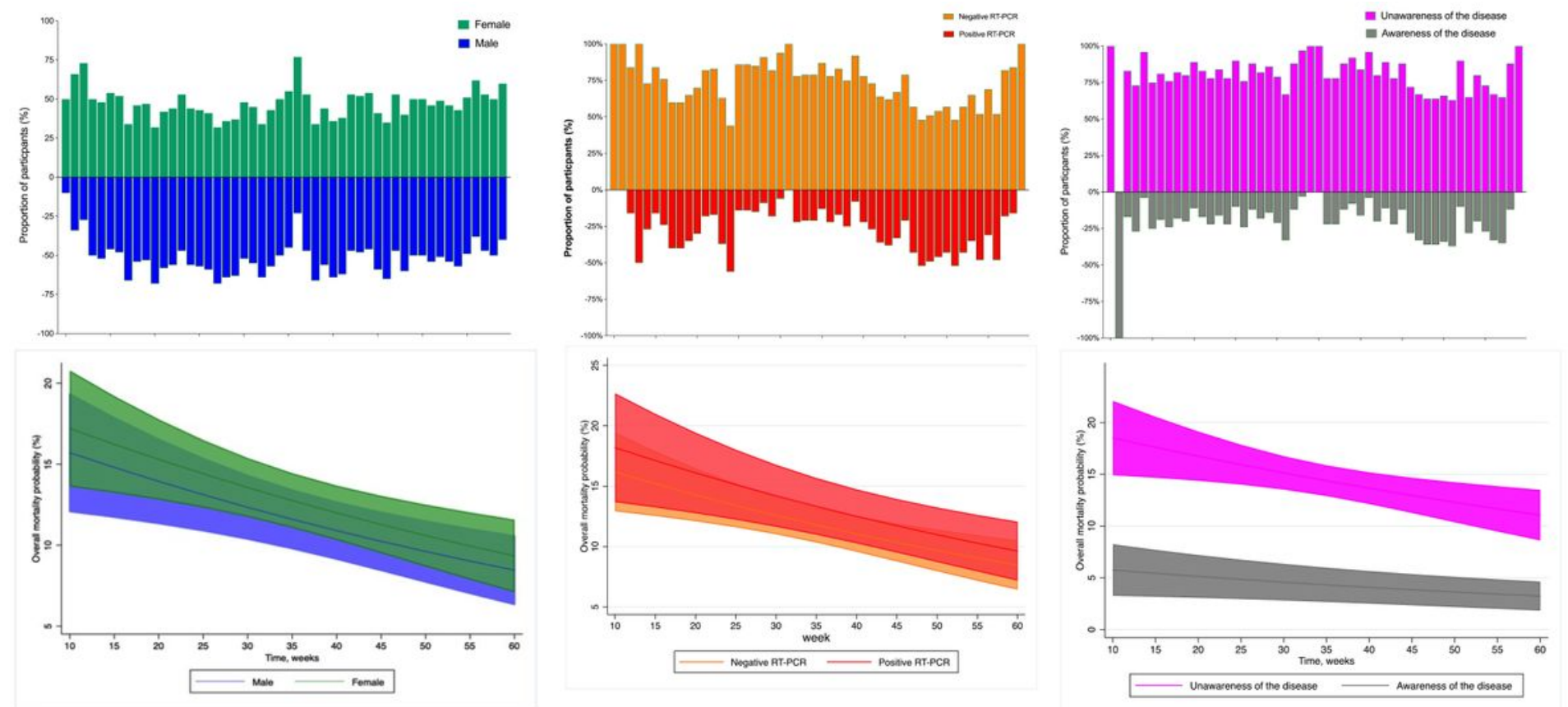

Figure 4

Proportion of COVID-19 patients admitted between March 9 and February 28 and overall mortality rate Stratified by (A) sex at birth, (B) RT-PCR, and (C) disease awareness. 


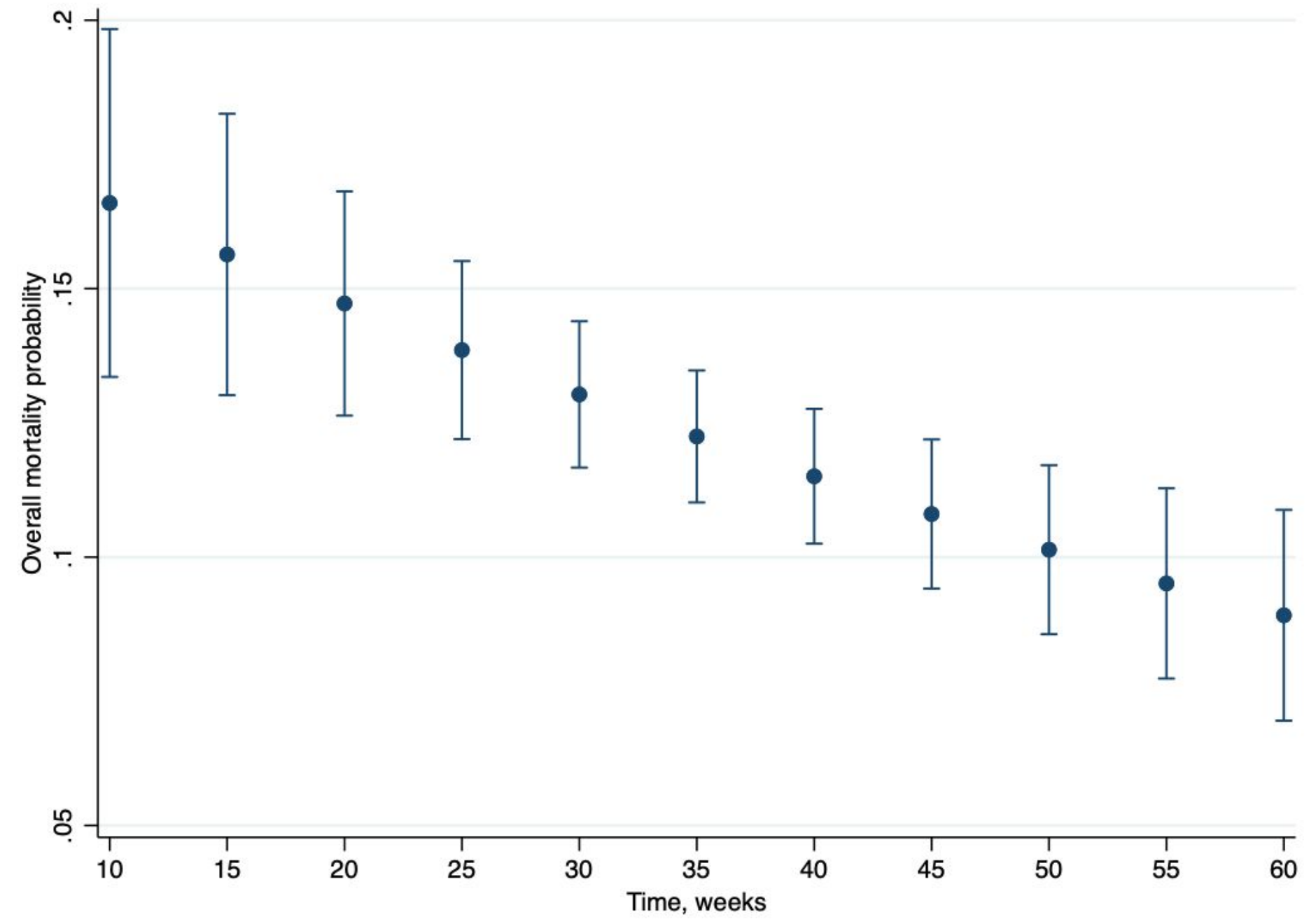

Figure 5

Weekly unadjusted mortality in patients admitted to the hospital with COVID-19 Error bars represent 95\% Cls.

\section{Supplementary Files}

This is a list of supplementary files associated with this preprint. Click to download.

- Additionalfile1.TimelineofinstitutionalchangesduringthefirstyearoftheCOVID19pandemic.pdf 